\title{
COMPONENTES GENÉTICOS EN POBLACIONES HETERÓTICAMENTE CONTRASTANTES DE MAÍZ DE ORIGEN TROPICAL Y SUBTROPICAL ${ }^{1}$
}

\author{
Ricardo Ernesto Preciado Ortiz ${ }^{2}$, Arturo Daniel Terrón Ibarra ${ }^{2}$, Noel Orlando Gómez Montiel ${ }^{2}$, Edgar Iván \\ Robledo González ${ }^{2}$
}

\begin{abstract}
RESUMEN
Componentes genéticos en poblaciones heteróticamente contrastantes de maíz de origen tropical y subtropical. Durante el ciclo O-I 02-03 se formó un dialélico con 16 poblaciones de origen tropical y subtropical. Las cruzas y sus progenitores fueron evaluados durante el ciclo P-V 2003 en el Campo Experimental Bajío- INIFAP. Con el objetivo de identificar las mejores poblaciones que permitan reforzar los programas de hibridación tanto en su estructura heterótica como en las fuentes de derivación de líneas. La información fue analizada mediante el método de Gardner y Eberhart con el cual, al separar los componentes de varianza, se estiman los efectos genéticos aditivos y no aditivos. Entre los resultados más relevantes se observó que no obstante la respuesta heterótica involucrada en la formación de estas poblaciones, el patrón heterótico "tropical x subtropical" se expresó en combinaciones específicas como PABGT x Sint "B"IG y Sint PABG I ACG x Sint “A”IG. con rendimientos de 12,5 y 12,4 t/ha respectivamente. En estos mismos cruzamientos se obtuvieron los máximos valores de heterosis específica que podría aprovecharse a través de Selección Recurrente Recíproca. La población Sint. "A" IG de origen tropical, presentó el mayor efecto varietal, y por su adaptación al subtrópico constituye un material prometedor para integrarlo al Programa de Mejoramiento Genético del Bajío. El mayor valor de heterosis varietal se observó en el sintético PABG I GH "A" lo cual es un indicador de Aptitud Combinatoria General que puede ser utilizado como probador. Un análisis más detallado, permitirá aprovechar este germoplasma en beneficio de los programas de mejoramiento genético de maíz.
\end{abstract}

Palabras clave: Patrones heteróticos; hibridación; cruzas dialélicas; germoplasma tropical x subtropical.

\begin{abstract}
Genetic components of maize heterotical contrasting tropical and subtropical populations. A diallelic cross between 16 tropical and subtropical populations was formed at Iguala, Gro., in the winter nursery 2002 - 2003. Crosses and parents were evaluated during the 2003 growing season at INIFAP Bajío Experimental Station, in Celaya, Gto, Mexico. The objective was to identify the best populations to support the heterotic structure in our breeding program and as sources to derived new inbred lines. Data were analyzed using the Gardner and Eberhart model, in which variance components can be separated to estimate additive and nonadditive genetic effects. Results showed that despite the heterotic response involved in the formation of the populations, the "tropical x subtropical" heterotic pattern was expressed in specific combinations such PABG T x Sint "B" IG and Sint PABG I ACG x Sint "A" IG, with grain yields of 12.5 and $12.4 \mathrm{t} / \mathrm{ha}$ respectively. These crosses presented maximum values of specific heterosis which can be used for developing high-yielding hybrids through reciprocal recurrent selection. Tropical population Sint "A" IG presented the highest varietal effect, and because of its good adaptation in the subtropic is considered an outstanding germplasm to be incorporated in the Bajío Maize Breeding Program. The best value of varietal heterosis, which is an indicator of General Combining Ability, was observed in PABG I GH "A" synthetic which can be used as tester. More detailed analysis will permit to take advantage of the germplasm involved to benefit maize breeding programs.
\end{abstract}

Key words: Heterotic patterns; hybridization; diallelic crosses; Tropical x Subtropical germplasm.

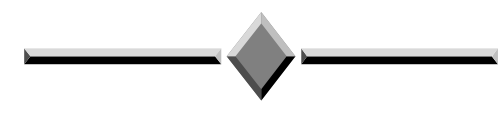

\footnotetext{
1 Recibido: 5 de mayo, 2005. Aceptado: 30 de agosto , 2005. Presentado en la LI Reunión Anual del PCCMCA. Panamá 2005.

2 Programa Nacional de Investigación en Maíz del INIFAP, con sede en los Campos Experimentales Bajío e Iguala. Correo electrónico: inifappreciado@prodigy.net.mx
} 


\section{INTRODUCCIÓN}

En México se siembran anualmente más de ocho millones de hectáreas con maíz, en una amplia diversidad de ambientes que van desde el nivel del mar hasta los valles altos (arriba de $2.200 \mathrm{msnm}$ ), con una amplitud de sistemas termo pluviométricos que van desde los muy drásticos hasta los muy benignos, y con esquemas de producción desde el auto abasto hasta altamente comercial. Debido a esta gran diversidad de "sistemas de producción", se estima que el uso de la semilla mejorada en México es menor al $25 \%$. Entre las alternativas para incrementar el uso de la semilla mejorada se encuentran a) Incrementar el potencial de rendimiento de los materiales mejorados, con miras a que sea económicamente viable el costo de la semilla; b) reducir los costos de la semilla mediante esquemas de autoabastecimiento de semilla por los productores; c) uso de híbridos varietales y no convencionales, en los cuales la semilla sea manejada como un recurso y no como un insumo.

Para dar respuesta a las alternativas anteriores, el Programa de Mejoramiento Genético de Maíz de El Bajío ha implementado, dentro de sus líneas de investigación, una serie de estrategias, como la estructuración de los esquemas de hibridación basados en la definición de patrones heteróticos, Terrón et al. (1997), Preciado et al. (2003) y Preciado-Ortíz y Johnson (2004).

La importancia de los patrones heteróticos ha sido presentada por Hallauer y coautores, Hallauer y Miranda (1988); Hallauer et al. (1988); Hallauer (1990) y Eyherabide y Hallauer (1991) y se resume en los siguientes puntos:

El establecimiento de los patrones heteróticos entre variedades es un factor clave en todos los programas de mejoramiento de maíz para seleccionar líneas endogámicas progenitoras de híbridos de alto potencial de rendimiento.

Las líneas derivadas de fuentes con patrones heteróticos definidos tienden a complementarse una con otra maximizando la respuesta heterótica del híbrido.

Los patrones heteróticos permiten la elección de probadores basada en el origen de las líneas.

También los mejoradores pueden aprovechar el conocimiento de la genealogía y su relación entre líneas al evaluar cruzas experimentales con líneas derivadas de patrones heteróticos definidos.

Después de haber establecido patrones heteróticos en un programa de mejoramiento, es posible optimizar e incrementar la respuesta heterótica a través de selección.
Los patrones heteróticos tienen fuerte impacto al ser considerados en esquemas de selección convencionales.

Basados en el concepto de patrón heterótico, recientemente en algunos programas de mejoramiento genético de maíz en México se han formado nuevas poblaciones sintéticas provenientes del reciclaje de germoplasma élite, clasificado por su respuesta heterótica. Estas poblaciones constituyen una nueva generación de germoplasma que pueden ser la fuente de derivación de líneas con mejores atributos agronómicos y mayor respuesta heterótica.

Con respecto a la utilidad de evaluar cruzas dialélicas, Comstock et al. (1949) señalan que de detectarse efectos mayores de la aptitud combinatoria general, es factible explotar la porción aditiva de la varianza genética disponible mediante cualquier variante de la selección recurrente; por el contrario en cruzamientos donde se registre mayor aptitud combinatoria específica puede implementarse un programa de selección recurrente recíproca, o de hibridación.

Al aplicar en dos grupos de distintas variedades el modelo estadístico propuesto por Gardner y Eberhart (1966), que a su vez es una modificación del diseño II de Griffing para análisis dialélicos, Terrón (1981) y Sierra y Preciado (1988) comentan que dicho modelo puede ser útil para obtener mayor conocimiento de la variación genética y aptitud combinatoria mediante los parámetros relacionados con la heterosis promedio, heterosis varietal y heterosis específica a partir de los cuales es posible detectar y seleccionar con un mayor grado de seguridad, tanto los genotipos más idóneos por mejorar, como la metodología más adecuada para realizar dicho mejoramiento.

Con base en lo anterior, el objetivo de este trabajo está enfocado a identificar las mejores poblaciones que permitan reforzar los programas de hibridación tanto en su estructura heterótica como en las fuentes de derivación de líneas.

\section{MATERIALES Y MÉTODOS}

Durante el ciclo otoño - invierno 02-03 se formaron cruzas dialélicas con poblaciones sintéticas provenientes del reciclaje de germoplasma élite, clasificado por su respuesta heterótica de origen tropical y subtropical. En todos los casos las poblaciones y sintéticos utilizados fueron de maíz blanco con textura que varía entre los tipos dentado y semicristalino. Las cruzas y sus progenitores fueron evaluados durante el ciclo primavera - verano 2003 en el Campo Experimental Bajío- INIFAP. En 
total se evaluaron 16 progenitoras de maíz con sus ((n x n-1) / 2) 120 cruzas dialélicas. Además de algunos testigos, cuya información no se incluye en este artículo. La genealogía de las poblaciones de referencia y su origen se presentan en el Cuadro 1.

Cuadro 1. Genealogía y origen de las poblaciones sintéticas de maíz utilizadas en la formación del dialélico analizado en este trabajo. Campo Experimental Iguala en el Ciclo otoño - invierno 2002-2003.

\begin{tabular}{cll}
\hline Orden & \multicolumn{1}{c}{ Genealogía } & Origen \\
\hline 1 & Comp.PABG-C & Subtropical \\
2 & Comp.PABG-C & Subtropical \\
3 & V 385 PB EB & Subtropical \\
4 & PABG-T & Subtropical \\
5 & Sint.PABG-T GH “A” & Subtropical \\
6 & Sint.PABG-T ACG & Subtropical \\
7 & Sint.PABG-I GH “A” & Subtropical \\
8 & Sint.PABG-I GH "B” & Subtropical \\
9 & Sint.PABG-I ACG & Subtropical \\
10 & V-322 E & Subtropical \\
11 & Sint. "A" IG & Tropical \\
12 & Sint. "B” IG & Tropical \\
13 & VS-535 & Tropical \\
14 & VS-536 & Tropical \\
15 & Pob. 345 & Subtropical \\
16 & PET & Tropical \\
\hline
\end{tabular}

El diseño experimental utilizado fue un latice simple de 12 x 12, con dos repeticiones, parcelas de dos surcos de $5 \mathrm{~m}$ de largo, la separación entre surcos fue de $0,75 \mathrm{~m}$ se sembró a una densidad de 80.000 ptas/ha y el manejo agronómico fue de acuerdo con lo recomendado por el Campo Experimental Bajío. En la conducción del trabajo de campo se buscó mantener en todos los tratamientos una fertilización y humedad uniforme.

Al obtener los datos ajustados del análisis de varianza, corregidos con base en el diseño de látice, se procedió al análisis del dialélico de acuerdo a la metodología de Gardner y Eberhart (1966), cuyo modelo es:

$$
Y_{j j},=M_{v}+1 / 2\left(V_{j}+V_{j},\right)+h_{j}{ }^{\prime}
$$

Donde:

$\mathrm{M}_{\mathrm{v}}=$ Media de las variedades progenitoras

$\mathrm{V}_{\mathrm{j}}=\mathrm{Al}$ efecto varietal de la $\mathrm{j}$-esima variedad ${ }^{2}$

$\mathrm{h}_{\mathrm{j} j} \mathrm{j}=$ Efecto de heterosis cuando la variedad $\mathrm{j}$ es cruzada con la variedad j'

\footnotetext{
2 Cuando se utiliza $\mathrm{V}_{\mathrm{j}}$, es con el fin de diferenciar los dos progenitores de una cruza, los cuales en los dos casos corresponden a una j-esima variedad.
}

El efecto de la heterosis se subdivide en:

$$
h_{j j},=h+h_{j}+h_{j}{ }^{\prime}+s_{j} j^{\prime}
$$

Donde:

$\mathrm{h}=$ Heterosis promedio

$h_{j}=$ Heterosis varietal contribuida por la variedad $\mathrm{j}$.

$\mathrm{h}_{\mathrm{j}}$, = Heterosis varietal contribuida por la variedad $\mathrm{j}$ '.

$s_{j} j^{\prime}=$ Heterosis específica debida al cruzamiento de las variedades $\mathrm{j}$ con $\mathrm{j}$ '.

Para expresar de manera cuantitativa los efectos que intervienen en el comportamiento de las variedades y cruzas en estudio, se estimaron valores para los efectos genéticos de acuerdo con Terrón (1981).

Efecto varietal $\left(\mathbf{V}_{\mathbf{j}}\right)$, calculado mediante la desviación de rendimiento varietal $\left(\hat{\mathrm{Y}}_{\mathrm{j}} \mathrm{j}\right.$ ) y la media de variedades $\left(\hat{\mathrm{Y}}_{\mathrm{v}}\right)$, con el siguiente modelo:

$$
\mathrm{V}_{\mathrm{j}}=\hat{\mathrm{Y}}_{\mathrm{j} j},-\hat{\mathrm{Y}}_{\mathrm{V}}
$$

Efecto de heterosis $\left(\mathbf{h}_{\mathbf{j}} \mathbf{j}\right.$ ) $)$ se calcula mediante la desviación de rendimiento varietal menos el promedio de rendimiento de los progenitores, cuyo modelo es:

$$
h_{j j},=Y_{j j},-1 / 2\left(Y_{j j}+Y_{j}{ }^{\prime}{ }^{\prime}\right)
$$

Heterosis promedio $(\hat{\mathbf{h}})$ se calcula por la diferencia entre la media de cruzas $\left(\hat{\mathrm{Y}}_{\mathrm{c}}\right)$ menos la media de variedades $\left(\hat{\mathrm{Y}}_{\mathrm{V}}\right)$, cuyo modelo es:

$$
\hat{\mathrm{h}}=\hat{\mathrm{Y}}_{\mathrm{c}}-\hat{\mathrm{Y}}_{\mathrm{v}}
$$

Heterosis varietal $\left(\mathbf{h}_{\mathbf{j}}\right)$ se calcula como la desviación de los progenitores $\left(\mathrm{Y}_{\mathrm{h}}\right)$ y los cruzamientos en que interviene cada variedad, de acuerdo al siguiente modelo:

$$
h_{j}=n-1 / n-2\left(\hat{Y}_{h}-\hat{Y}_{c}\right)-1 / 2\left(Y_{j j},+Y_{v}\right)
$$

Heterosis específica $\left(\mathbf{S}_{\mathbf{j j}}\right)$, calculada a partir de la desviación de la cruza y el promedio de todas las cruzas por el promedio de cada progenitor; ilustra la aptitud combinatoria específica de las cruzas estudiadas, de acuerdo al siguiente modelo:

$$
S_{j j},=Y_{j j},+(n / n-2) Y_{c}-n-1 / n-2\left(\hat{Y}_{h}+\hat{Y}_{h},\right)
$$

\section{RESULTADOS Y DISCUSIÓN}

La información presentada a continuación son los resultados del análisis e interpretación del dialélico entre poblaciones sintéticas progenitoras (Variedades), 
únicamente para la variable rendimiento de grano expresado en $\mathrm{kg} / \mathrm{ha}$. En el Cuadro 2 se presenta el análisis de varianza de los efectos genéticos que influyen en la expresión del material utilizado. Se observaron diferencias altamente significativas para todos los componentes de variación, por lo que se puede decir que todos ellos influyen en la expresión del rendimiento. La significancia del factor materiales pudo deberse a la gran amplitud de variación para rendimiento entre las poblaciones y las variedades y/o las cruzas estudiadas, que osciló entre 4.759 a 12.530 kg/ha (Cuadro 3). Además al descomponer este factor de variación entre variedades y cruzas, ambas presentaron diferencias altamente significativas que sugieren que tanto las variedades como las cruzas influyen de manera importante al rendimiento.
Bajo el supuesto de que el factor variedad representa la porción genética aditiva, y el factor cruzas la no aditiva, se puede interpretar que tanto en las variedades como en las cruzas estudiadas existen considerables efectos aditivos y no aditivos que pueden ser explotados tanto por esquemas de selección recurrente, como recurrente recíproca y de hibridación, para capitalizar ambos efectos en las poblaciones como lo sugiere Comstock et al. (1949).

Respecto a la descomposición del factor de variación cruzas en los diferentes tipos de heterosis, se observó diferencias significativas en los tres componentes, heterosis promedio, heterosis varietal, y heterosis específica. Esta respuesta positiva en los tres componentes

Cuadro 2. Análisis de varianza de los efectos genéticos, de los datos obtenidos en el Campo Experimental Bajío en primavera verano 2003

\begin{tabular}{lrrrl}
\hline Factor de variación & G.L. & S. C. & C. M. & Fc \\
\hline Materiales & 135 & 249933256 & $1851357,5^{* *}$ & 0,02155431 \\
Variedades & 15 & 79489668,96 & $5299311,3^{* *}$ & 0,0616969 \\
Cruzas & 120 & 170443587,5 & $1420363,2^{* *}$ & 0,01653649 \\
Het. promedio & 1 & 43301819,69 & $43301819,7^{* *}$ & 0,50413872 \\
Het. varietal & 15 & 19906083,8 & $1327072,3^{* *}$ & 0,01545036 \\
Het. específica & 104 & 107235684 & $1031112,3^{* *}$ & 0,01200466 \\
Error & 135 & 11595510174 & 85892668,0 & \\
\hline
\end{tabular}

** Altamente significativo al 0,01 de probabilidad.

Cuadro 3. Rendimiento de grano ( $\mathrm{kg} / \mathrm{ha}$ ) del dialélico de poblaciones intermedias-tardías, datos obtenidos en el Campo Experimental Bajío en Primavera Verano 2003.

\begin{tabular}{|c|c|c|c|c|c|c|c|c|c|c|c|c|c|c|c|c|c|}
\hline Poblaciones & 1 & 2 & 3 & 4 & 5 & 6 & 7 & 8 & 9 & 10 & 11 & 12 & 13 & 14 & 15 & 16 & $\overline{\mathbf{Y}}_{\mathbf{h}}$ \\
\hline 1 Comp.PABG-C & 6.425 & 7.209 & 7.465 & 8.481 & 8.333 & 8.758 & 8.931 & 8.587 & 7.975 & 8.740 & 11.096 & 8.719 & 7.831 & 9.075 & 9.084 & 7.644 & $8.528,53$ \\
\hline 2 Comp.PABG-C & & 6.737 & 9.868 & 8.479 & 7.435 & 8.281 & 7.200 & 8.852 & 7.200 & 10.086 & 8.300 & 8.607 & 7.552 & 7.354 & 8.849 & 6.864 & $8.142,40$ \\
\hline 3 V 385 PB EB & & & 8.161 & 9.430 & 9.641 & 8.756 & 11.648 & 9.271 & 9.722 & 7.896 & 8.501 & 9.570 & 10.202 & 9.0531 & 10.777 & 8.762 & $9.370,80$ \\
\hline 4 PABG-T & & & & 8.479 & 9.014 & 9.363 & 8.715 & 7.897 & 8.553 & 9.292 & 11.705 & 12.530 & 9.088 & 9.305 & 9.161 & 9.404 & $9.361,13$ \\
\hline 5 Sint.PABG-T GH “A” & & & & & 7.419 & 9.146 & 10.789 & 8.190 & 11.547 & 9.287 & 11.603 & 11.462 & 8.608 & 10.196 & 7.929 & 8.699 & $9.458,60$ \\
\hline 6 Sint.PABG-T ACG & & & & & & 6.311 & 9.7911 & 10.103 & 9.352 & 9.192 & 9.715 & 9.834 & 10.233 & 8.930 & 9.440 & 7.236 & $9.208,67$ \\
\hline 7 Sint.PABG-I GH “A” & & & & & & & 4.759 & 9.571 & 7.112 & 10.147 & 9.898 & 11.943 & 9.739 & 9.169 & 9.427 & 8.558 & $9.509,20$ \\
\hline 8 Sint.PABG-I GH "B" & & & & & & & & 8.767 & 9.007 & 8.322 & 10.440 & 9.351 & 9.782 & 10.4021 & 10.418 & 9.857 & $9.336,67$ \\
\hline 9 Sint.PABG-I ACG & & & & & & & & & 6.660 & 9.706 & 12.404 & 9.391 & 9.017 & 9.900 & 9.417 & 8.245 & $9.236,53$ \\
\hline $10 \mathrm{~V}-322 \mathrm{E}$ & & & & & & & & & & 10.393 & 11.049 & 9.953 & 11.039 & 11.159 & 9.857 & 9.243 & $9.664,53$ \\
\hline 11 Sint. "A" IG & & & & & & & & & & & 10.941 & 9.771 & 7.834 & 11.601 & 9.482 & 8.8351 & $10.148,93$ \\
\hline 12 Sint. "B" IG & & & & & & & & & & & & 8.571 & 8.139 & 9.008 & 8.944 & 8.468 & $9.712,67$ \\
\hline 13 VS-535 & & & & & & & & & & & & & 6.031 & 7.738 & 9.017 & 8.821 & $8.976,00$ \\
\hline 14 VS-536 & & & & & & & & & & & & & & 7.403 & 9.627 & 8.154 & $9.378,07$ \\
\hline 15 Pob. 345 & & & & & & & & & & & & & & & 7.109 & 10.033 & $9.430,80$ \\
\hline 16 PET & & & & & & & & & & & & & & & & 5.865 & $8.588,20$ \\
\hline
\end{tabular}


de variación, permite por un lado corroborar el valor de las variedades estudiadas a través de su heterosis promedio. Aprovechar la aptitud combinatoria general estimada mediante la heterosis varietal, y aprovechar la heterosis específica para la formación de híbridos de alto potencial de rendimiento derivados de combinaciones específicas.

En el Cuadro 3 se presentan los rendimientos de las variedades progenitoras así como de las cruzas donde se pueden destacar las combinaciones especificas de PABGT x Sint "B” IG y Sint. PABGI ACG x Sint "A" IG rendimientos de 12.530 y $12.404 \mathrm{~kg} / \mathrm{ha}$ respectivamente.

En el Cuadro 4 se presenta el efecto varietal $\mathrm{Vj}$ donde el Sint."A" IG y V 322 fueron los progenitores de mayor rendimiento, sin embargo, como se verá más adelante, al analizar su comportamiento mediante sus cruzas con otros materiales, el Sint." A" IG mantuvo un gran efecto de heterosis varietal $\left(h_{j}\right)$ no así el V $322 \mathrm{cu}$ yo efecto fue negativo. El Sint."A" IG al ser una variedad de origen tropical y por su buena adaptación al subtrópico, constituye un material prometedor para integrarlo al programa de mejoramiento genético de maíz de El Bajío.

Respecto al efecto de heterosis $\left(\mathrm{h}_{\mathrm{ji}}\right)$ que se presenta en el Cuadro 5, se observa que existen cruzas individuales tales como Sint PABGI GH "A" x Sint "B" IG y V 385 PB EB x Sint PAGB I GH "A” que mostraron los mayores valores de heterosis. Sin embargo, aprovechando la
Cuadro 4. Efecto varietal $(\mathrm{Vj})$ de las poblaciones intermediastardías estudiadas, datos obtenidos en el Campo Experimental Bajío en primavera - verano 2003.

\begin{tabular}{rlr}
\hline Variedad & \multicolumn{1}{c}{ Genealogía } & Vj \\
\hline 11 & Sint.”A” IG & 3439 \\
10 & V-322 E & 2891 \\
8 & Sint.PABG-I GH “B” & 1265 \\
12 & Sint.”B” IG & 1069 \\
4 & PABG-T & 977 \\
3 & V 385 PB EB & 659 \\
5 & Sint.PABG-T GH “A” & -83 \\
14 & VS-536 & -99 \\
15 & Pob. 345 & -393 \\
2 & Comp.PABG-C & -765 \\
9 & Sint.PABG-I ACG & -842 \\
1 & Comp.PABG-C & -1077 \\
6 & Sint.PABG-T ACG & -1191 \\
13 & VS-535 & -1471 \\
16 & PET & -1637 \\
7 & Sint.PABG-I GH “A” & -2743 \\
\hline
\end{tabular}

ventaja de la separación de los efectos de heterosis, será posible una mejor interpretación de dicho efecto.

El valor de heterosis promedio (h) fue de 1.751 $\mathrm{kg} / \mathrm{ha}$; aunque hubo algunos casos donde los cruzamientos no superaron al rendimiento de sus progenitores.

Cuadro 5. Valores del efecto de heterosis (hjj') de las cruzas estudiadas, datos obtenidos en el Campo Experimental Bajío en primavera - verano 2003.

\begin{tabular}{|c|c|c|c|c|c|c|c|c|c|c|c|c|c|c|c|c|}
\hline Poblaciones & 1 & 2 & 3 & 4 & 5 & 6 & 7 & 8 & 9 & 10 & 11 & 12 & 13 & 14 & 15 & 16 \\
\hline 1 Comp.PABG-C & & 628 & 172 & 1.029 & 1.411 & 2.390 & 3.339 & 991 & 1.433 & 331 & 2.413 & 1.221 & 1.603 & 2.161 & 2.317 & 1.499 \\
\hline 2 Comp.PABG-C & & & 2419 & 871 & 357 & 1.757 & 1.452 & 1.100 & 502 & 1.521 & -539 & 953 & 1.168 & 284 & 1.926 & 563 \\
\hline 3 V 385 PB EB & & & & 1.110 & 1.851 & 1.520 & 5.188 & 807 & 2.312 & -1.381 & -1.050 & 1.204 & 3.106 & 1.271 & 3.142 & 1.749 \\
\hline 4 PABG-T & & & & & 1.065 & 1.968 & 2.096 & -726 & 984 & -144 & 1.995 & 4.005 & 1.833 & 1.364 & 1.367 & 2.232 \\
\hline 5 Sint.PABG-T GH “A” & & & & & & 2.281 & 4.700 & 97 & 4.508 & 381 & 2.423 & 3.467 & 1.883 & 2.785 & 665 & 2.057 \\
\hline 6 Sint.PABG-T ACG & & & & & & & 4.256 & 2.564 & 2.867 & 840 & 1.089 & 2.393 & 4.062 & 2.073 & 2.730 & 1.148 \\
\hline 7 Sint.PABG-I GH "A" & & & & & & & & 2.808 & 1.403 & 2.571 & 2.048 & 5.278 & 4.344 & 3.088 & 3.493 & 3.246 \\
\hline 8 Sint.PABG-I GH "B" & & & & & & & & & 1.294 & -1.258 & 586 & 682 & 2.383 & 2.317 & 2.480 & 2.541 \\
\hline 9 Sint.PABG-I ACG & & & & & & & & & & 1.180 & 3.604 & 1.776 & 2.672 & 2.869 & 2.533 & 1.983 \\
\hline $10 \mathrm{~V}-322 \mathrm{E}$ & & & & & & & & & & & 382 & 471 & 2.827 & 2.261 & 1.106 & 1.114 \\
\hline 11 Sint. "A" IG & & & & & & & & & & & & 15 & -652 & 2.429 & 457 & 432 \\
\hline 12 Sint. "B" IG & & & & & & & & & & & & & 838 & 1.021 & 1.104 & 1.250 \\
\hline 13 VS-535 & & & & & & & & & & & & & & 1.021 & 2.447 & 2.873 \\
\hline 14 VS-536 & & & & & & & & & & & & & & & 2.371 & 1.520 \\
\hline 15 Pob. 345 & & & & & & & & & & & & & & & & 3.546 \\
\hline $16 \mathrm{PET}$ & & & & & & & & & & & & & & & & \\
\hline
\end{tabular}


Respecto a la heterosis varietal (hj) que como se mencionó antes, puede ser un indicador de aptitud combinatoria general; por lo que puede permitir identificar genotipos para utilizarlos como "probadores". En el Cuadro 6, se puede observar que el Sint PABGI GH "A" presentó el mayor valor en este efecto, no obstante que dicho sintético estaba clasificado como grupo heterótico "A", la explicación de esta respuesta es por un lado

Cuadro 6. Efectos de heterosis varietal (hj), datos obtenidos en el Campo Experimental Bajío en Primavera-Verano 2003.

\begin{tabular}{rlr}
\hline Variedad & \multicolumn{1}{c}{ Genealogía } & \multicolumn{1}{c}{ hj } \\
\hline & & \\
7 & Sint.PABG-I GH “A” & 1.645 .72 \\
6 & Sint.PABG-T ACG & 547,72 \\
13 & VS-535 & 438,43 \\
9 & Sint.PABG-I ACG & 403,08 \\
15 & Pob, 345 & 386,72 \\
5 & Sint.PABG-T GH “A” & 261,50 \\
14 & VS-536 & 183,22 \\
16 & PET & 105,93 \\
12 & Sint.”B” IG & $-42,28$ \\
3 & V 385 PB EB & $-203,57$ \\
1 & Comp.PABG-C & $-238,00$ \\
4 & PABG-T & $-372,92$ \\
8 & Sint.PABG-I GH “B” & $-543,14$ \\
11 & Sint.”A” IG & $-759,85$ \\
2 & Comp.PABG-C & $-807,71$ \\
10 & V-322 E & $-1.004,85$ \\
\hline
\end{tabular}

que fue la variedad que presentó el menor rendimiento per se $(4.759 \mathrm{~kg} / \mathrm{ha})$ y por el otro en todos los casos sus cruzas superaron el rendimiento del progenitor.

La heterosis específica $\left(\mathrm{S}_{\mathrm{jj}}\right.$ ) que ilustra la aptitud combinatoria específica, se presenta en el Cuadro 7 donde los máximos valores ocurrieron en los cruzamientos PABGT x Sint "B" IG y Sint PABGI ACG x Sint "A" IG, en ambas cruzas se observa la presencia del patrón heterótico Tropical x Subtropical. El aprovechamiento de estas cruzas puede ser de manera directa a través de la comercialización de la cruza intervarietal como tal para sistemas de producción donde se requiera semilla de bajo costo; también con la derivación de líneas de dichas variedades será posible la obtención de híbridos de alto potencial de rendimiento; y finalmente con los progenitores de estas cruzas podría implementarse un esquema de selección recurrente recíproca.

\section{CONCLUSIONES}

De las poblaciones evaluadas el Sint "A" IG presentó el mayor valor de efecto varietal, por lo que se considera la más adecuada para aprovechar la porción aditiva de la varianza genética a través de selección recurrente, además de su utilización como tal, y la derivación de líneas para la formación de híbridos.

Los mayores efectos de heterosis fueron observados en las cruzas Sint PABGI GH "A" x Sint "B" IG y V 385 PB EB x Sint PABGI GH “A”.

Cuadro 7. Valores de heterosis específica ( $\left.\mathrm{S}_{\mathrm{jj}}{ }^{\prime}\right)$ de las cruzas estudiadas, datos obtenidos en el Campo Experimental Bajío en Primavera Verano 2003.

\begin{tabular}{|c|c|c|c|c|c|c|c|c|c|c|c|c|c|c|c|c|}
\hline Poblaciones & 1 & 2 & 3 & 4 & 5 & 6 & 7 & 8 & 9 & 10 & 11 & 12 & 13 & 14 & 15 & 16 \\
\hline 1 Comp.PABG-C & & $-77,6$ & $-1.137,7$ & $-111,4$ & $-363,8$ & 329,0 & 180,0 & 20,8 & $-483,9$ & $-177,4$ & $1.659,6$ & $-250,0$ & $-348,7$ & 464,5 & 417,0 & $-120,2$ \\
\hline 2 Comp.PABG-C & & & $1.679,0$ & 300,3 & $-848,1$ & 265,7 & $-1.137,3$ & 699,6 & $-845,2$ & $1.582,3$ & $-722,7$ & 51,7 & $-214,0$ & $-842,8$ & 595,7 & $-486,5$ \\
\hline 3 V 385 PB EB & & & & $-64,8$ & 41,8 & $-575,4$ & $1.994,6$ & $-1.97,6$ & 360,7 & $-1.923,9$ & $-1.837,9$ & $-301,4$ & $1.119,8$ & $-459,9$ & 1207,6 & 95,3 \\
\hline 4 PABG-T & & & & & $-574,9$ & 41,9 & $-928,1-$ & $-1.561,2$ & $-797,9$ & $-517,5$ & $1.376,5$ & $2.668,9$ & 16,2 & $-197,6$ & $-398,1$ & 747,7 \\
\hline 5 Sint.PABG-T GH “A” & & & & & & $-279,5$ & $1.041,5-$ & $-1.372,7$ & $2.091,6$ & $-626,9$ & $1.170,1$ & $1.496,5$ & $-568,2$ & 589,0 & $-1734,5$ & $-61,7$ \\
\hline 6 Sint.PABG-T ACG & & & & & & & 311,3 & 808,1 & 164,4 & $-454,2$ & $-450,2$ & 136,3 & $1.324,6$ & $-409,2$ & 44,3 & $-1256,9$ \\
\hline 7 Sint.PABG-I GH “A” & & & & & & & & $-45,9-$ & $-2.397,6$ & 178,8 & $-589,2$ & $1.923,3$ & 508,6 & $-492,2$ & $-290,7$ & $-256,9$ \\
\hline 8 Sint.PABG-I GH "B" & & & & & & & & & $-317,7$ & $-1.461,3$ & 137,7 & $-483,9$ & 736,4 & 925,6 & 885,1 & $1.226,9$ \\
\hline 9 Sint.PABG-I ACG & & & & & & & & & & 30,0 & $2.209,0$ & $-336,6$ & 78,7 & 530,9 & $-8,6$ & $-277,8$ \\
\hline $10 \mathrm{~V}-322 \mathrm{E}$ & & & & & & & & & & & 395,4 & $-233,2$ & $1.642,1$ & $1.331,3$ & $-27,2$ & 261,6 \\
\hline 11 Sint. "A" IG & & & & & & & & & & & & $-934,2$ & $-2.081,9$ & $1.254,3$ & $-921,2$ & $-665,4$ \\
\hline 12 Sint."B" IG & & & & & & & & & & & & & $-1.309,4$ & $-871,2$ & $-991,7$ & $-564,9$ \\
\hline 13 VS-535 & & & & & & & & & & & & & & $-1.351,9$ & $-129,4$ & 577,3 \\
\hline 14 VS-536 & & & & & & & & & & & & & & & 49,8 & $-520,4$ \\
\hline 15 Pob. 345 & & & & & & & & & & & & & & & & 1302,1 \\
\hline $16 \mathrm{PET}$ & & & & & & & & & & & & & & & & \\
\hline
\end{tabular}


El Sint PABGI GH “A” fue el que presentó los valores más altos de heterosis varietal, debido al bajo rendimiento per se.

Las cruzas PABGT x Sint "B" IG y Sint PABGI ACG x Sint "A" IG mostraron la mayor aptitud combinatoria específica representada por la heterosis específica.

El patrón heterótico de germoplasma Tropical $\mathrm{x}$ Subtropical, se manifestó de manera importante en los cruzamientos de este estudio.

\section{LITERATURA CITADA}

COMSTOCK, R.E.; ROBINSON, M.F; HARVEY, P.H. 1949. A breeding procedure design to make maximum of both general and specific combining ability. Agron. J. 41: $360-367$.

EYHERABIDE, C.H.; HALLAUER, A.R. 1991. Reciprocal full sib recurrent selection in Maize: II Contribution of additive, dominance and genetic drift effects. Crop Science 31: 1442-1448.

GARDNER, C.O.; EBERHART, S.A. 1966. Analysis and interpretation of the variety cross diallel and related populations. Biometrics 22: 439 - 452.

HALLAUER, A.R.; MIRANDA FO., J.B. 1988. Quantitative genetics in maize breeding. Iowa State University Press. USA. 468 p.
HALLAUER, A.R; RUSSELL, W.A.; LAMKEY, K.R. 1988. Corn breeding. In: G.F. Sprague; J.W. Dudley eds. Corn and Corn Improvement Agron. Monogr: No. 18. ASA,CSSA, and SSSA. Madison WI. USA. p. 463-564.

HALLAUER, A.R. 1990. Methods using in developing maize inbreeds. Maydica 35:1-16.

PRECIADO, R.E.; TERRÓN, A.D.; GÓMEZ N.O.; SIERRA, M.; ERAZO, M. 2003. Use of heterotic pattern concept to group inbred lines from different sources. Arnel $\mathrm{R}$ Hallauer International Symposium on Plant Breeding. Mexico D.F. p. 276-277.

PRECIADO-ORTÍZ. R.E; JOHNSON, B.E. 2004. Potential use of maize heterotic patterns for grouping and adapting exotic germplasm. Maydica 49: 199-208.

TERRÓN, A.D. 1981. Análisis e interpretación de cruzas dialélicas en variedades tropicales de maíz adaptadas a Nayarit. Tesis Profesional. Escuela de Agricultura. Universidad de Guadalajara, Jal. México. 52 p.

TERRÓN, A.D.; PRECIADO, R.E.; PONS, J.L. 1997. Utilization of heterotic patterns to develop maize hybrids for El Bajío. The genetic and explotation of heterosis in crops. International Simposium. Mexico D.F. p 357.

SIERRA, M.; PRECIADO, R.E. 1988. Análisis e interpretación de cruzas dialélicas con germoplasma tropical precoz de maíz. Rev. Fitotec. Mex. 11: 92 -102. 